Relations industrielles

Industrial Relations

\title{
Trade Union Democracy in Western Europe, par Walter Galenson, University of California Press, Berkeley and Los Angeles, 1961, 97 pp.
}

\section{Gérard Dion}

Volume 16, numéro 4, octobre 1961

URI : https://id.erudit.org/iderudit/1021690ar

DOI : https://doi.org/10.7202/1021690ar

Aller au sommaire du numéro

\section{Éditeur(s)}

Département des relations industrielles de l’Université Laval

ISSN

0034-379X (imprimé)

1703-8138 (numérique)

Découvrir la revue

Citer ce compte rendu

Dion, G. (1961). Compte rendu de [Trade Union Democracy in Western Europe, par Walter Galenson, University of California Press, Berkeley and Los Angeles, 1961, 97 pp.] Relations industrielles / Industrial Relations, 16(4), 507-508.

https://doi.org/10.7202/1021690ar

Tous droits réservés (C Département des relations industrielles de l’Université Laval, 1961
Ce document est protégé par la loi sur le droit d'auteur. L’utilisation des services d'Érudit (y compris la reproduction) est assujettie à sa politique d'utilisation que vous pouvez consulter en ligne.

https://apropos.erudit.org/fr/usagers/politique-dutilisation/ 
and, when opportune, even upon all the members of the political community, since all, in the final analysis, benefit by such changes of automation. This can the more surely be obtained when the workers, through their unions and organizations, are present and have a voice in the implementation of processes of automation.

It is obvious that workers will be able to assume responsibilities in production processes when they are properly prepared to do so, that is, when they are professionally instructed and socially educated. The schools can and must make their contribution to this; but an equally important contribution can be made by the workers themselves through the wise utilization of their free time; and appropriate institutions promoted by common accord between workers and their organizations on the one hand, and management and direction on the other, can also contribute validly and usefully. In this regard, the Pontife's exhortation in \& Mater et Magistra should be borne in mind: «In social education the Associations and Organizations of the Lay Apostolate play an important role, especially those that have as their specific objective the Christianization of the economic and social sectors of the temporal order. Indeed, many members of these Associations can draw profit from their daily experiences to form themselves more completely and also to contribute to the social education of youth $\$$.

\section{RECENSIONS}

Trade Union Democracy in Western Europe, par Walter Galenson, University of California Press, Berkeley and Los Angeles, 1961, 97 pp.

L'auteur, qui a déjà dirigé la publication d'un ouvrage en collaboration, Comparative Labor Movements, nous donne ici une description sommaire de la structure et du fonctionnement des syndicats ouvriers dans certains pays d'Europe occidentale: Italie, France, Belgique, Hollande, Autriche, Grande-Bretagne et pays scandinaves. Comme le centre d'intérêt est de découvrir le genre de démocratie qui existe dans ces groupements, il est naturellement amené à vérifier jusqu'à quel point on rencontre les critères qui sont souvent utilisés pour établir la démocratie syndicale: concurrence syndicale, participation des membres à la vie syndicale, affiliation obligatoire, répartition de l'autorité entre la centrale nationale, les fédérations et des groupements locaux, etc.

Il ressort de cette étude que le syndicalisme ouvrier, comme les autres institutions, est essentiellement un produit de la culture, de l'histoire, du régime économique et politique d'un pays déter- miné. Il existe, sans doute, des phénomènes qui se trouvent partout. Ainsi, par exemple, une certaine désaffection vis-à-vis du syndicalisme à mesure que celui-ci atteint une certaine maturité. Toutefois, les mêmes causes produisent des effets différents dans des contextes différents. Le pluralisme syndical peut, à la fois, être élément de faiblesse ou de puissance syndicale, comme il peut aussi contribuer à la démocratie syndicale autant qu'à la concentration du pouvoir entre les mains des dirigeants. \&We must guard against any erroneous postulation of a necessary one-to-one relationship between union democracy and union rivalry $»$. Structures et pratiques syndicales ne sont pas des denrées exportables.

Il reste, cependant, que Pétude comparative des divers mouvements syndicaux est utile pour mieux faire comprendre le syndicalisme que nous avons ici, en Amérique. L'auteur, d'ailleurs, y fait des références constantes. Nous croyons que tous ceux qui s'arrêtent à vouloir repenser notre syndicalisme auraient grand intérêt à connaître cet ouvrage. De même encore, tous ceux qui sont portés à juger sommairement une 
institution aussi complexe.

L'étude du professeur Galenson est de nature à rendre service au syndiealisme et à la démocratie.

\section{GÉRARD Dion}

Essays in Labour History, in Memory of G.D.H. Cole, edited by Asa Briggs and John Saville, with recollections of G.D.H. Cole by Ivor Brown, Hugh Gaitskell, Stephen K. Bailey and G.N.D. Worswick; London, Macmillan and Co. Ltd., New York, St. Martin's Press, 1960.

Voici un ouvrage qui plaira sûrement aux admirateurs de l'historien des classes laborieuses britanniques, au penseur et au professeur hors pair que fut G.D.H. Cole, et qui intéressera au plus haut point les spécialistes en histoire du travail. Ce recueil d'essais auquel ont collaboré un certain nombre d'universitaires anglais, est précédé d'une série de témoignages personnels sur Cole émanant d'anciens condisciples, d'anciens élèves ou de collègues d'université qui ont eu l'avantage de travailler avec lui ou de le voir à l'oeuvre à différents stages de sa brillante carrière.

Ce livre, qui devait être présenté à Cole à l'occasion de son soixante-dixième anniversaire de naissance, est devenu, du fait de la mort de ce dernier, un tribut à sa mémoire et à l'influence qu'il a exercé sur sa génération.

Les quarante premières pages du volume sont donc consacrées au retracement de la carrière de Cole, au collège, puis à l'université comme étudiant d'abord, puis comme professeur, homme de pensée, écrivain et homme d'action. En même temps que les traits dominants de sa personnalité, les témoignages qui nous sont offerts, font ressortir, dans un langage mêlé d'admiration et de respect, les hautes qualités du professeur, du penseur et de l'animateur que fut Cole aux yeux de l'intelligentsia socialiste britannique de la première moitié du vingtième siècle: les souvenirs de Hugh Gaitskell nous révèlent ce qui, d'après lui, a fait que Cole, tout en étant parmi les plus brillants représentants de l'authentique pensée socialiste en Angleterre, n'a jamais ou presque, été amené à faire de la politique active. Ceux de G.D.N. Worswick nous font saisir l'universalité de l'esprit de Cole, qui, à ses yeux, fut tout à la fois sociologue, politicologue, économiste et historien, en plus de me- ner de front une foule d'activités dans l'ordre universitaire et social et ceci, en dépit d'une santé toujours chancelante. Ce qui ressort de ces témoignages, c'est la profondeur et l'envergure de la connaissance chez Cole, alliées à une capacité de travail et de rayonnement par la parole et par l'écrit qui tient du prodige.

Le reste du volume, c'est-à-dire quelque trois cents pages, est consacré aux essais mentionnés plus haut. Ces essais ont ceci en commun qu'ils portent tous, à divers titres, sur l'histoire du mouvement du travail dans l'Angleterre du dixneuvième siècle, un des sujets qui ont intéressé le plus Cole en tant qu'historien. Les sujets traités comprennent l'évolution du terme «classe 》 au cours des premières décennies du XIXe siècle; la croissance et les différentes réalisations du mouvement coopératif britannique en partant de Robert Owen et de son «socialisme 》 communautaire jusqu'aux visées plus réalistes des pionniers de Rochdale et des institutions coopéra. tives qu'ils ont inspirées par la suite: le régime des salaires et en général l'analyse des marchés du travail dans l'économie libérale de Ricardo, Mill et des classiques anglais, y compris les attitudes et les politiques d'action des trade-unions en matière de revendication dans un tel régime; des monographies d'épisodes marquants qui ont jalonné l'histoire du socialisme britannique des années 1860 et suivantes; deux essais biographiques, l'un sur Beesly et son «ultra-radicalisme $\gg$ et l'autre sur Tom Maguire et l'Independant Labour Party de la fin du XIXe siècle et des débuts du XXe siècle; un essai est consacré à l'historique, en Angleterre, de la première Internationale; enfin, une excellente revue des évènements qui conduisirent à la fameuse décision judiciaire connue sous le nom de «Taff Vale Case» et dans laquelle on met en lumière les menées antisyndicales qui se firent jour de plus en plus dès la fin de la grève des dockers de Londres en 1889 pour aboutir à la condamnation, par les tribunaux, des droits acquis statutairement quelque vingt-cinq ans auparavant, vient clore cette série d'études.

Ce volume reprend donc «en gros plan » si l'on peut dire, quelques scènes historiques de l'histoire sociale britannique et, à ce titre, ne peut manquer d'être utile à ceux qui sont déjà familiers avec les traités plus généraux qui existent en cette matière.

Jean-Réal Cardin 